\title{
GRACE/GRACE-FO Observed Mass Change Patterns of Antarctica Extracted by ICA Method
}

\author{
Tianyan Shi ${ }^{1}$, Yoichi Fukuda ${ }^{2}$, Koichiro Doi ${ }^{2}$ and Jun 'ichi Okuno ${ }^{2}$ \\ ${ }^{1}$ The Graduate University for Advanced Studies, SOKENDAI, Kanagawa, Japan \\ ${ }^{2}$ National Institute of Polar Research, Tokyo, Japan
}

Antarctica is one of the most rapidly mass changing areas with poor ground observation data on the Earth. Considering the dominant uncertainty in Glacial Isostatic Adjustment (GIA) effects for the Antarctic Ice Sheet (AIS) mass balance, we analyzed mass change patterns of Antarctica qualitatively using a statistics-based blind source separation method named Independent Component Analysis (ICA). We extracted the 6 leading independent components of mass change in equivalent water height (E.W.H) using gravimetric data derived from the Gravity Recovery and Climate Experiment (GRACE) mission and GRACE Follow-On (GRACE-FO) mission (Figures 1 and 2). We used the latest spherical harmonic coefficients published by three official data centers (JPL, CSR, GFZ). The results showed that the whole continental mass changes could be effectively separated into several spatial patterns that would be dominated by different physical processes as follows:

Mode 1 (IC1) showing a clear linear trend which can be found on some well-known regions with intense glacial melting signals such as the Amundsen Sea and Bellingshausen Sea sector of the West AIS and Totten of East AIS, as well as the mass increasing regions like Dronning Maud Land of East AIS, suggested a relation with ice sheet mass changes,

Mode 2 (IC2) showing mild mass loss regions distributing over the Antarctic continent and a relatively small amplitude and high-frequency signals in time-series, suggested a relation with the meteorological phenomena,

Mode 3 (IC3) showing another linear trend component different from Mode 1 and the mass increases mainly distributed in Marie Byrd Land of West Antarctica, suggested a relation with the GIA effects,

Mode 4 (IC4) showing the mass changing of Palmer Land of Antarctic Peninsula, and a relatively long period time changes, suggested a relation with a periodic climate variation,

Mode 5 (IC5) showing the mass increases in East Antarctica with leaps and bounds around 2009, suggested a relation with the abnormal snowfall event, and

Mode 6 (IC6) showing the mass increases mainly distributed in Ronne Ice Shelf and continent of East Antarctica and a mixture of a long-term trend and high-amplitude cyclical signal in time-series, suggested a mixture of the meteorological variations and other phenomena.

Although the hidden independent physical processes cannot be completely separated, some significant signals such as glacial melting components, snow accumulation components, periodic climatic components, and GIA effects could be determined without introducing any external information.

We also found that the period of the employed data sets has a direct impact on ICA results. This suggested that some impacts of extreme events, such as rapid increases of snowfall in the late 2000s might cause dramatic changes spatially and temporally. In general, ICA provides a special view for a better understanding of AIS mass changes and specific regional researches. 

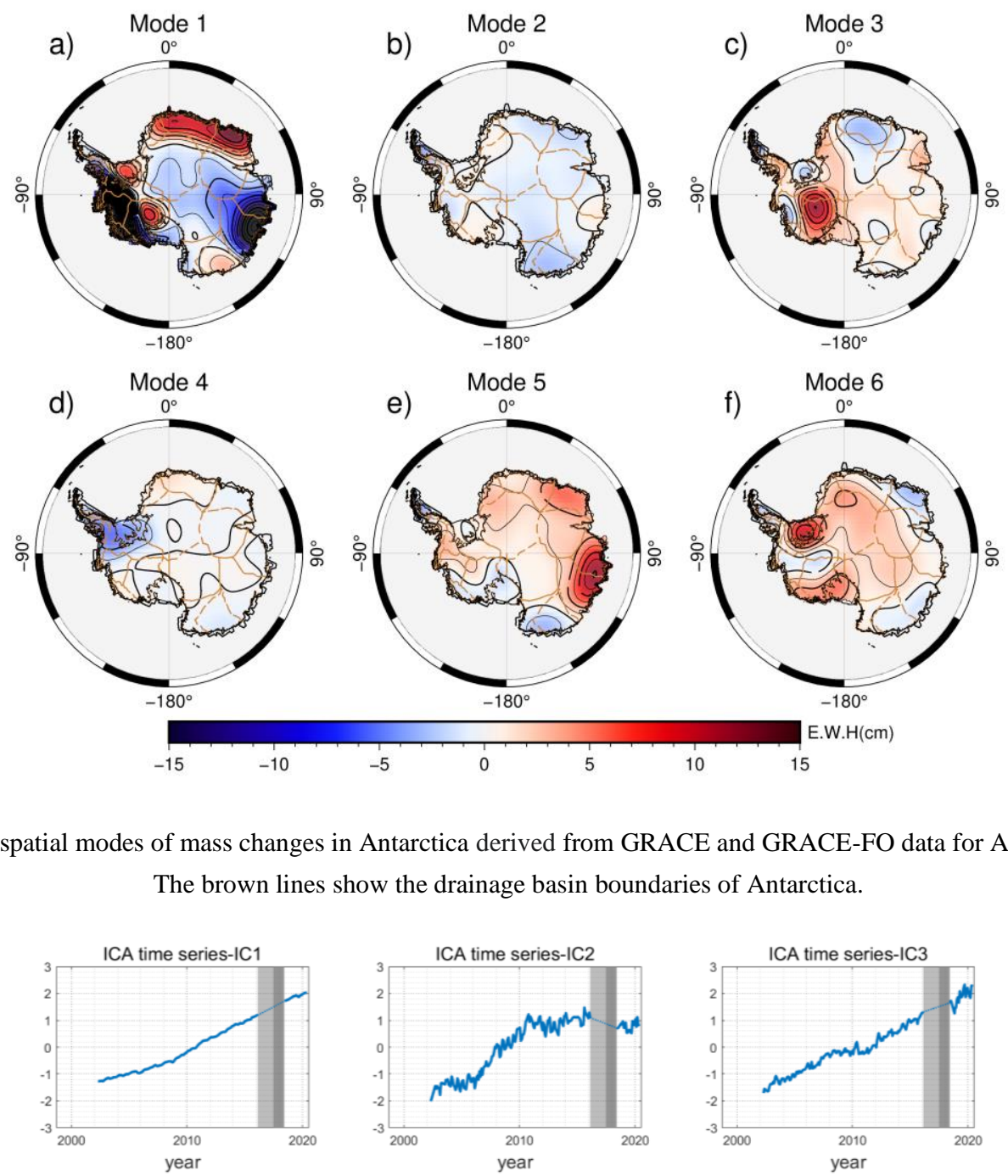

Figure 1. The first six spatial modes of mass changes in Antarctica derived from GRACE and GRACE-FO data for April 2002 to June 2020. The brown lines show the drainage basin boundaries of Antarctica.

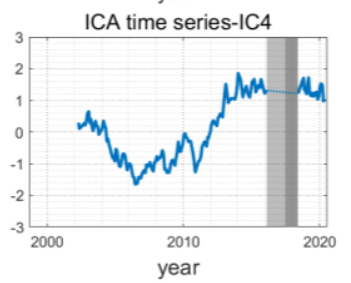

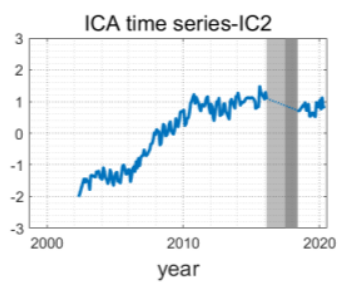

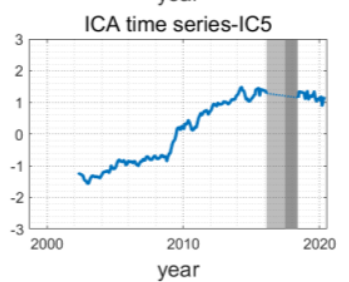

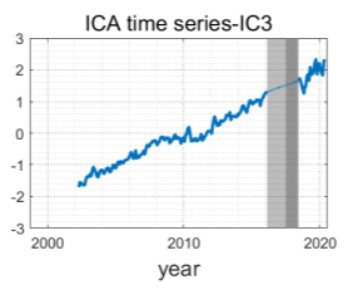

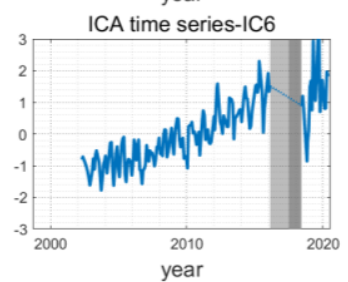

Figure 2. Corresponding time series of six spatial modes. The Gray bar indicates the data absent period, the dark bar indicates the GRACE/GRACE-FO data gap from July 2017 to April 2018, the light bar indicates an extra one year of data discarded near the ending of the GRACE mission due to the deteriorating of GRACE observation. 